\title{
Der Kopf des Magnus Maximus
}

\author{
Francis X. Ryan
}

Gratian wurde im Jahre 383 n. Chr. „auf der Flucht in Lyon getötet.“1 Hieronymus berichtet darüber in einer im Jahre 396 verfassten Schrift. Nach ihm wurden die Bewohner von Lyon noch 13 Jahre nach dem Mord an jenes Verbrechen erinnert: Gratianus ab exercitu suo proditus et ab obviis urbibus non receptus ludibrio hosti fuit cruentaeque manus vestigia parietes tui, Lugdune, testantur (Hieron. Ep. 60.15.3). Bei der Wiedergabe des Satzes ist durchweg von einer blutigen Hand die Rede. Der fragliche Satzteil wird etwa von F. A. Wright (Loeb: New York 1933) wie folgt übersetzt: ,your walls, O Lyons, still bear the mark of that bloody hand." Erst vor wenigen Jahren wurde die herkömmliche Interpretation von D. Woods neu gedeutet. Er stellte die Frage in den Mittelpunkt, wem die Hand zuzuschreiben wäre. Da das Wort „bloody“ eine Gewalttat evoziere, jedoch keine Quelle eine solche dem Kaiser zuschreibt, wäre die Hand nicht auf ihn zu beziehen. Anstatt die Hand als die des Mörders des jungen Kaisers anzusehen, zog Woods in Betracht, dass die fragliche manus nicht als eine menschliche Hand zu deuten sei: „It is difficult to understand what it actually means to say that either Gratian or his enemy left the traces of his bloody hand upon the walls. On the face of it, this seems to require that the individual concerned left a bloody hand-print on the wall." ${ }^{2}$ Woods legte eine andere Definition des Wortes manus zugrunde: „I would like to propose an alternative translation and interpretation..., that manus here means 'band' rather than 'hand'... In brief, this passage refers to the ancient practice by which emperors placed the heads of those whom they had had executed as public enemies on public display." "3 Woods erinnert zu Recht, dass Theodosius I. im Jahre 388 Magnus Maximus, Gratians Gegenkaiser, drei Meilen entfernt von Aquileia (Cons. Constant. a. 388), in Norditalien, köpfen ließ (Claud. IV Cons. Hon. 85, Philost. HE 10.8) und dass keine Quelle glaubwürdig überliefert, was mit dessen Kopf geschah.

Einem Fragment Olympiodors zufolge wurde zwar der Kopf des Maximus, wie fünf andere auch (Olymp. fr. 20.1Bl=Phot. Bibl. cod. 80) in Karthago zur Schau gestellt, man darf allerdings von einem Überlieferungsfehler in der Nennung von $\mathrm{K} \alpha \varrho \theta \alpha \gamma \varepsilon \dot{v} \eta\rceil$ statt $\mathrm{P} \alpha \beta \varepsilon \dot{v} v \vee \eta \varsigma$ ausgehen. ${ }^{4}$ Erhärtet wird die Annahme eines Fehlers dadurch, dass die abgeschlagenen Köpfe des Iovinus (411-413) und seines Bruders Sebastian (412-413) gemäß der Lokalchronik der Stadt (Ann. Rav. a. 412) am 30. August 412 dorthin gebracht wurden; die Behauptung in einer Anfang des 9. Jhdts. verfassten Weltchronik (Theoph. Chron. AM 5904), dass die Köpfe nach Rom gebracht worden wären, ist nicht vorzuziehen. Laut dem Fragment Olympiodors waren Constantin III. (407-411) und sein Sohn Iulianus, wie vorher bereits Maximus (383-388) und Eugenius (392-394), dort enthauptet worden, wo die Köpfe von Iovinus und Sebastian gezeigt wurden. Woods hält die Nachricht über Constantin und seinen Sohn für glaubwürdig, da sich Constantin erst im Jahre 407 zum Gegenkaiser des Honorius erhob und Ravenna ab dem Jahr 402 die Hauptstadt war. Nach Woods ist der Fall von Maximus und Eugenius jedoch anders zu bewerten. Beide ließen sich während der Regierungszeit des Theodosius I. zu Gegenkaisern proklamieren, wie das Fragment Olympiodors auch ausdrücklich belegt, und Mailand fungierte $\mathrm{zu}$ dem

\footnotetext{
${ }^{1}$ Lippold 1980, 34.

${ }^{2}$ Woods 1999, 56.

${ }^{3}$ Woods 1999, 57.

${ }^{4}$ Vgl. Blockley 1983, 216 A. 50.
} 
Zeitpunkt als Hauptstadt. Woods mag mit der Vermutung richtig gelegen haben, dass Photios eine Aussage über das gleiche Verfahren unter Theodosius dahingehend missverstand, dass es auch am gleichen Ort angewandt wurde, ${ }^{5}$ denn Olympiodor selbst war über die Geschehnisse im Westen des Reiches gut informiert. ${ }^{6}$ Woods zog aber nicht den Schluss, dass der Kopf des Maximus nach Mailand gesandt wurde, obwohl nahegelegt wird, dass der Kopf des Eugenius dort zu sehen war: „Indeed, one doubts whether these two heads alone even need have been put on display in the same place.“7 Dem Fragment Olympiodors glaubte Woods entnehmen zu können, „that

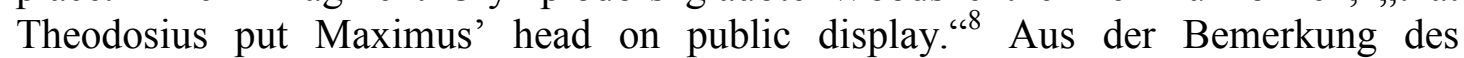
Hieronymus folgerte er aber, ,that Theodosius sent the heads of Maximus and of some of his chief supporters to the city-walls of Lyons as their final resting-place.... One envisages a line of heads mounted upon stakes set upon the walls of Lyons...."

Woods ging nicht auf eine andere Quelle (Cons. Constant. a. 411: Constantini tyranni in conto caput adlatum est XIIII kal. Octob.) ein, der zufolge der Kopf des Constantin III. nach Spanien geschickt wurde. Diese Nachricht erhöht allerdings die Wahrscheinlichkeit seiner Rekonstruktion. Woods argumentierte, dass Theodosius die Köpfe von Maximus und einigen seiner Anhänger nach Lugdunum schickte, ,and that he did so in memory of Gratian whom they had allegedly had killed nearby and whose murder he claimed to be avenging. "“10 Honorius schickte seinerseits den Kopf des in Britannien zum Kaiser ausgerufenen Constantin III. nach Spanien, weil dieser dort zwei seiner Verwandten, die Brüder Didymus und Verenianus, wegen ihres Widerstandes hatte töten lassen (vgl. Olymp. fr. 17.1Bl). ${ }^{11}$ Die Entscheidung, den abgeschlagenen Kopf eines Gegners sozusagen an den Tatort zu bringen und dort ausstellen zu lassen, ist für einen der unmittelbaren Nachfolger des Theodosius belegt, und die Möglichkeit, dass der Kopf des Maximus in Lugdunum ausgestellt war, ist nicht von der Hand zu weisen.

Gegen die Rekonstruktion von Woods erheben sich jedoch mehrere Einwände:

1. Es ist nicht als selbstverständlich zu betrachten, dass der Kopf des Magnus Maximus in Lugdunum permanent ausgestellt hätte werden sollen. Maximus wurde in Britannien zum Kaiser ausgerufen; wenn der Zweck darin bestand, Nachahmungstäter abzuschrecken, dann wäre der Kopf in Britannien besser aufgehoben gewesen. Angesichts der Tatsache, dass die Colonia Augusta Treverorum als Residenzstadt des Maximus fungierte ${ }^{12}$ und früher Gratian ebenfalls als Residenz gedient hatte, ${ }^{13}$ hätte Theodosius den Kopf auch dort ausstellen lassen können.

Zudem wissen wir nicht, ob die Bestrafung des Constantin III. einen Parallelfall bietet. Es steht fest, dass Maximus allenfalls der Auftraggeber des Mordes war und sich zur fraglichen Zeit nicht am Todesort aufhielt. Vor Ort war damals Andragathius, der Kommandeur der Kavallerie; ${ }^{14}$ er hatte sich nach der Niederlage

\footnotetext{
${ }^{5}$ Woods 1999, 58.

${ }^{6}$ Rohrbacher 2002, 77-81.

${ }^{7}$ Woods 1999, 58.

${ }^{8}$ Woods 1999, 58.

${ }^{9}$ Woods 1999, 58-59.

${ }^{10}$ Woods 1999, 58 u. A. 13; er zweifelte, dass Maximus und seine Vertrauten den Mord anordneten, ,though it served Theodosian propaganda afterwards to claim that they had.“

${ }^{11}$ Dazu s. Seeck 1900, 1029.

${ }^{12}$ Enßlin 1935, 2548.

${ }^{13}$ Vgl. Seeck 1913, 166.

${ }^{14}$ In der PLRE (1.62) wird er als Magister Equitum des Magnus Maximus eingestuft.
} 
des Maximus von seinem Schiff ins Meer gestürzt ${ }^{15}$ und war gleichsam außerhalb der Reichweite der Justiz. Constantin III. hingegen dürfte am Geschehen direkter beteiligt gewesen sein als dies bei Maximus der Fall war.

Es ist bedauerlich, dass wir nicht mehr über die Bestrafung des Eugenius wissen, denn hier liegt der gleiche Tatbestand vor: Der romanisierte Franke Arbogast galt in der offiziellen Version als der Mörder des jungen Kaisers Valentinian II., der Usurpator Eugenius seinerseits lediglich als der Anstifter des Mordes (Claud. IV cons. Hon. 75-76). Gratians Halbbruder wurde im Mai 392 in seinem Palast in Vienna erhängt aufgefunden. Wenn feststünde, daß Theodosius später den Kopf des Eugenius nach Vienna schickte, dann würden wir mit ziemlicher Sicherheit wissen, daß er früher den des Maximus nach Lugdunum sandte.

2. Es ist nicht nachgewiesen, ob es Brauch war, den Kopf eines Usurpators permanent auszustellen; wenn nicht, dann können die vestigia des Briefes unmöglich die Köpfe von Maximus und seinen Anhängern sein, denn der Brief wurde erst etwa acht Jahre nach der Niederlage des Maximus verfasst. Woods räumte ein, ,the evidence does not confirm...how long...these heads were left on display," fand es aber „consistent with the very purpose of such behaviour to assume that the heads were eventually set on semi-permanent display somewhere after the initial parades and festivities of abuse. "16 Man darf allerdings bezweifeln, dass der Schädel, der nicht mehr als der Kopf des Usurpators zu erkennen war, ausgestellt blieb. Caesar bekam seinerzeit lediglich den Kopf und den Ring (Vir. ill. 77.9: caput...cum anulo) des Pompeius präsentiert, und der Kopf war nicht zuletzt darum in ein Tuch eingehüllt (ebd.: caput...Aegyptio velamine involutum), weil er erkennbar bleiben sollte.

3. Auch wenn man einräumt, dass der Kopf des Maximus nach Lugdunum gebracht worden und dort jahrelang ausgestellt geblieben sein könnte, ist die Übersetzung eines Menschenschädels mit vestigia zu hinterfragen. Woods wurde möglicherweise durch das englische Wort „vestige“ verleitet, was ,a small, degenerate, or rudimentary organ or part" bedeutet. ${ }^{17}$ Zwar kann das Wort vestigia die übertragene Bedeutung „Ruinen“ haben (Lewis-Short, Oxford 1879, s. v.), wie in der Wendung semiruta murorum vestigia (Amm. 24.2.6), es war aber jedenfalls nicht üblich, mit diesem Wort sterbliche Überreste zu bezeichnen. Bei letzteren würde man eher das Wort reliquiae erwarten (Lewis-Short, s. v.: „the remains, relics, ashes of a deceased person; esp. of a body that has been burned"). Die eigentliche Bedeutung von vestigium ist ,a footstep“ (Lewis-Short, s. v.), so dass das damit Bezeichnete nur schwerlich ein Teil eines Ganzen sein kann. Wie der Fußstapfen lediglich der Abdruck eines Fußes ist, nicht der Fuß selbst oder eine Zehe, dürfen die vestigia das sein, was ein Ereignis nur indirekt bestätigt. Körperteile scheinen als Erklärung auszuscheiden.

Woods geht nicht darauf ein, warum Hieronymus die Schädel nicht capita nennt. Es ließe sich argumentieren, dass die Schädel, die nicht nur Sonne, Regen und Wind, sondern auch den Vögeln ausgesetzt waren, nicht mehr ganz erhalten waren und deshalb nicht capita genannt wurden. Aber auch Schädel, denen etwa die Unterkiefer fehlten, wären wohl viel zu gut erhalten gewesen, um als vestigia gegolten zu haben. Der Brief des Hieronymus an Heliodorus, den Bischof von Altinum, ${ }^{18}$ war freilich ein ambitioniertes literarisches Unterfangen - es handelt sich um ein Trostschreiben zum Tode von Nepotianus, dem Neffen des Bischofs - , man

\footnotetext{
15 Seeck 1894, 2132.

${ }^{16}$ Woods 1999, 57 A. 7.

${ }_{17}^{17}$ Siehe beispielshalber The American Heritage Dictionary of the English Language, Boston 1969, s. v.

${ }^{18}$ Zur Person s. Larue 1995, 1406.
} 
muss daher mit der Möglichkeit rechnen, dass der Autor ein alltägliches Wort wie capita umschreiben wollte. Das Wort caput besitzt jedoch wie im Deutschen „Haupt“ und im Englischen „head“ eine Zweideutigkeit, es kann nämlich sowohl „Haupt“ als auch „Hauptperson“" bedeuten (Lewis-Short, s. v.). Der Interpretation Woods folgend ließe sich zugleich von den „Köpfen“ bzw. „Schädeln“ und den „Anführern“ der Bande sprechen. Es fällt schwer zu glauben, dass sich Hieronymus ein anderes Wort ausgesucht hätte, wenn er an der fraglichen Stelle von den Schädeln der bewussten Anführer hätte sprechen wollen.

4. Woods beachtete nicht weiter, dass sich durch seine Interpretation von manus die Bedeutung des Wortes cruentae verschiebt: weder nahm er Anstoß an der Übersetzung des Wortes cruentae mit „bloody“ noch erwähnte er das Wort cruentae, als er seine neue Übersetzung für das Wort manus vorschlug. Eine „bloody hand“ ist „blutig“, eine „bloody band“ ist ebenfalls „blutig“, man kann sie aber auch als „,bloodthirsty“ bzw. „,blutrünstig“ bezeichnen. Für das Adjektiv cruentus werden beide Bedeutungen aufgeführt; der eigentlich Sinn ist „mit Blut befleckt“, der übertragene Sinn „blutdürstig“ (Lewis-Short, s. v.). Die Übersetzung von cruentae manus mit „der blutrünstigen Bande" ist zwar nicht falsch, jedoch mag sie in diesem konkreten Fall nicht korrekt sein. Die Sinnverschiebung des Wortes cruentae hat zur Folge, dass die Mauer frei von Blut war. Es regt sich der Verdacht, dass Hieronymus den Usurpator und seine Anhänger anders beschrieben hätte, wenn nur deren Schädelknochen auf der Mauer zu sehen gewesen wären. Dieses Bauwerk zeigte (parietes...testantur) etwas; eine Mauer ist aber ein stummer Zeuge, sie kann kaum eine Charaktereigenschaft wie Blutrünstigkeit belegen, ohne weiteres aber eine eingetrocknete Flüssigkeit zeigen. Man will die wohl unerklärliche Übersetzung „die Abdrücke einer blutigen Hand" nicht wieder beleben, man könnte aber aus der Kollokation von cruentae und vestigia schließen, dass an dieser Stelle von Blutflecken die Rede ist. Blutflecken sind nicht nur etwas, was eine Mauer zeigen (testantur) kann, sondern auch etwas, was im Lateinischen mit vestigia ausgedrückt werden konnte, denn wie Fußabdrücke sind Blutflecken Zeichen.

5. Die Pluralform vestigia scheint Woods veranlasst zu haben, die Strafe auf einige Gefolgsmänner des Maximus auszudehnen. Wenn es überhaupt richtig wäre, vestigium in der Singularform als einen Knochen aufzufassen, dann könnte man trotzdem unter vestigia den Schädel eines einzigen Menschen verstehen, denn der Unterkiefer, der bei Totenschädeln häufiger fehlt, lässt sich leicht von den anderen Schädelknochen unterscheiden.

6. Der Brauch, aufgespießte Menschenschädel auf einer Stadtmauer aufzustellen, ist tatsächlich allein durch die Trajanssäule für die Daker bezeugt. ${ }^{19}$

Nach den Schlachten bei Siscia und Poetovio ergab sich Magnus Maximus den Offizieren des Theodosius; vor letzteren, der sich drei Meilen vor der Stadt Aquileia einquartiert hatte, wurde er am 28. August 388 (Cons. Constant. a. 388, Fasti Vind. pr. a. 388) geführt und anschließend geköpft. Theodosius hielt sich im darauf folgenden Winter in Mailand auf; ${ }^{20}$ wenn der Kopf dorthin gesandt wurde, dann könnte Olympiodor dies in seinem Originaltext richtig festgestellt haben. Dass man später den Kopf des Constantin III. nicht am Hinrichtungsort hinterließ, erscheint plausibel: Er und sein jüngerer Sohn wurden 30 Meilen vor Ravenna enthauptet (Olymp. fr. 17.1Bl) und Honorius war in der Hauptstadt anwesend.

\footnotetext{
${ }^{19}$ Lepper-Frere 1988, P1. XX (Scene XXV), m. S. 72: „skulls set on tall poles“; aufgespießte, auf einer Mauer aufgestellte Köpfe sind nicht belegt.

${ }^{20}$ Gibbon-Milman 1846, 539.
} 
Es sind keine Schriften überliefert, wie Theodosius mit dem Kopf des Eugenius verfuhr. Jedoch ist der gleiche Tatbestand wie bei der ersten Usurpation festzustellen: Im September 394 war Theodosius bei der Schlacht am Frigidus anwesend, in deren Laufe Eugenius gefangen und geköpft (Philost. HE 11.2, Sok. HE 5.25, Soz. HE 7.24) wurde. In diesem Fall ist überliefert, was unmittelbar danach geschah: Der abgeschlagene Kopf des Eugenius, aufgespießt auf einer Lanze, wurde im Lager herumgetragen, um die überlebenden Soldaten des Eugenius zu bekehren (Zos. 4.58.5). Wenn man mit Woods dem Fragment Olympiodors entnimmt, dass der Kopf des Maximus an einem nicht bekannten Ort ausgestellt war, fiele die erste Wahl auf Mailand. Die Aussage über den Hinrichtungsort scheint allerdings die eigene Zutat des Photios zu sein, und dies lässt eher vermuten, dass bei Olympiodor selbst kein Ausstellungsort für den Kopf des Maximus angegeben war.

Gibt es Grund zur Annahme, dass Theodosius den Kopf des Maximus überhaupt zur Schau stellen wollte? Nach der Schlacht bei Lugdunum schickte Septimius Severus den Kopf seines Gegenkaisers Clodius Albinus nach Rom (Dio Epit. 76.7.3, Hdn. 3.8.1): Führende Senatoren hatten mit Albinus in Korrespondenz gestanden und ihn gebeten, nach Rom zu kommen, als Septimius Severus im Osten des Reiches den Kampf gegen Pescennius Niger noch austrug (Hdn. 3.5.2). Septimius Severus wollte also vor allem die Senatoren einschüchtern. In einem Brief an das Volk erklärte er seine Entscheidung, den Kopf des Albinus in Rom öffentlich auszustellen; aus dem überlieferten Text, der einige Schwierigkeiten bereitet, geht trotzdem klar genug hervor, dass er von Zorn (Hdn. 3.8.1: ỏ@ үَv) erfüllt war. Nach seinem Sieg über Maximus soll Theodosius dagegen seinen Zorn beherrscht haben (Pacat. 45.4: tu...omnem cum armis iram deposivisti). Diese Aussage in der Rede, die etwa ein Jahr nach der Enthauptung des Maximus ${ }^{21}$ im Senat in Anwesenheit des Kaisers gehalten wurde, scheint die Zurschaustellung des Kopfes des Besiegten vollends auszuschließen. Zieht man indes die Eigentümlichkeiten dieser Textgattung in Betracht, so könnte man vermuten, dass der Lobredner hier eine Floskel benutzt. Es heißt, Theodosius habe darüber nachgedacht, die Hinrichtung auszusetzen, doch seien die Seinen ihm zuvorgekommen (\$44.2: quin iam coeperas de eius morte dubitare et deieceras oculos.... sed...tui te vindicant et invitum. rapitur...et...inter innumeras manus fertur ad mortem). Nach Pacatus konnte Theodosius kaum ertragen, das Ende des Maximus auch nur erzählt $\mathrm{zu}$ bekommen (\$44.3: ecce iterum, imperator, averteris, et illam tyrannici exitus relationem gravaris). Er versicherte dem Kaiser, dass er keine Angst zu haben brauche (ebd.: iam, iam esto securus): Was er nicht ansehen wollte, werde er sich auch nicht anhören (ebd.: geram clementiae tuae morem: quod noluisti videre non audies). Die Worte des Pacatus beweisen nicht, dass Theodosius mitfühlend war, sie verraten uns aber, dass ihm trotzdem daran lag in der öffentlichen Meinung für einen mitfühlenden Menschen gehalten zu werden. ${ }^{22}$ Diese Hoffnung des Kaisers setzt voraus, dass es zu einer öffentlichen Zurschaustellung des Kopfes in einer der Großstädte des Reiches eben nicht kam. Der Kopf des Maximus wurde daher vermutlich weder in Karthago noch in Ravenna noch in Lugdunum noch in Mailand noch in Rom ausgestellt.

\footnotetext{
${ }^{21}$ Zur Datierung s. Nixon-Rodgers 1994, 443-444.

${ }^{22}$ Vgl. Nixon 1994, 513 A. 164 (ad §46): "Pacatus seems to have been well informed about Theodosius' attitudes."
} 


\section{Verzeichnis der abgekürzt zitierten Literatur}

\section{Blockley 1983}

Blockley, R. C.: The Fragmentary Classicising Historians of the Later Roman Empire, vol. 2, Liverpool 1983.

Enßlin 1935

Enßlin, W.: Maximus 33, in: RE 14 (1935), 2546-55.

Gibbon-Milman 1846

Gibbon, E., und H. H. Milman: The History of the Decline and Fall of the Roman Empire, 2 ${ }^{\text {nd }}$ ed., vol. 2, London 1846.

Jones-Martindale-Morris 1971

Jones, A. H. M., J. R. Martindale, und J. Morris: The Prosopography of the Later Roman Empire, Vol. 1: A.D. 260-395, Cambridge 1971.

Larue 1995

Larue, P.: Heliodorus, in: LThK 4 (1995), 1406.

Lepper-Frere 1988

Lepper, F., and S. Frere: Trajan's Column: A New Edition of the Cichorius Plates, Gloucester 1988.

Lippold 1980

Lippold, A.: Theodosius der Große und seine Zeit, 2. Aufl., München 1980.

Nixon-Rodgers 1994

Nixon, C. E. V., and B. S. Rodgers: In Praise of Later Roman Emperors: The Panegyrici Latini, Berkeley 1994.

Rohrbacher 2002

Rohrbacher, D.: The Historians of Late Antiquity, London 2002.

Seeck 1894

Seeck, O.: Andragathius 1, in: RE 1 (1894), 2132.

Seeck 1900

Seeck, O.: Constantin 5, in: RE 4 (1900), 1028-31.

Seeck 1913

Seeck, O.: Geschichte des Untergangs der antiken Welt, Bd. 5, Berlin 1913.

Woods 1999

Woods, D.: Jerome, Ep. 60.15.3: Gratian and the Walls of Lyons, in: AHB 13 (1999), 56-59. 